\title{
Clinical, endoscopic and histopathological evaluation of the efficacy of budesonide in the treatment of inflammatory bowel disease in dogs
}

\author{
A. Rychlik, A. Kołodziejska-Sawerska, M. Nowicki, M. Szweda \\ Department of Clinical Diagnostics, Faculty of Veterinary Medicine, \\ University of Warmia and Mazury in Olsztyn, Oczapowskiego 14, 10-719 Olsztyn, Poland
}

\begin{abstract}
This study evaluates the efficacy of budesonide in the treatment of inflammatory bowel disease (IBD) in dogs based on the results of clinical, endoscopic and histopathological examinations. The severity of clinical symptoms was assessed based on CIBDAI scores, and macroscopic and histopathological changes were described in accordance with the recommendations of the WSAVA Gastrointestinal Standardization Group for 2008. The results of the experiment revealed that budesonide does not offer effective treatment for canine IBD. The tested drug failed to alleviate the clinical symptoms of disease, lower CIBDAI scores, or improve the macroscopic appearance of intestinal mucosa. The effectiveness of budesonide was most highly evaluated in the histopathological picture of duodenal, jejunal and colonic mucosa.
\end{abstract}

Key words: IBD, budesonide, dogs, CIBDAI, endoscopy

\section{Introduction}

Glucocorticosteroids (prednisone, prednisolone) presently constitute the most effective treatment for severe inflammatory bowel disease (IBD) in dogs (CIBDAI scores higher than 9 points) (Malewska et al. 2011, Simpso-n and Jergens 2011). The mechanism of glucocorticosteroid activity in the treatment of IBD has not been fully elucidated. Glucocorticosteroids probably exert anti-inflammatory effects by inhibiting the release of inflammatory mediators and suppressing reactions that lead to the production of cytokines (Angelucci et al. 2008). Despite their therapeutic benefits, glucocorticosteroids carry serious side effects during long-term administration, including iatrogenic hyperadrenocorticism and suppression of the hypothalamic-pituitary-adrenal (HPA) axis (Tumulty et al. 2004). The use of glucocorticosteroids in dogs may also be limited due to the risk of adverse side effects, such as polyuria, polydipsia, polyphagia, hyperventilation, urinary tract infections, muscular dystrophy and steroid hepatopathy (Tumulty et al. 2004, Malewska et al. 2011).

In recent years, non-systemic glucocorticosteroids with low bioavailability have been increasingly often used in the treatment of Crohn's disease in humans. 
These drugs were designed to minimize side-effects while maintaining their therapeutic benefits (Campieri et al. 1997, Tumulty et al. 2004). The most popular non-systemic glucocorticosteroid is budesonide. In humans, budesonide is characterized by high levels of local anti-inflammatory activity in the intestinal lumen and considerably weaker systemic effects than traditional glucocorticosteroids (Tumulty et al. 2004). (Craven et al. 2004, Jergens and Simpson 2012).

There is an absence of published research investigating the influence of new generation glucocorticosteroids, including budesonide, on the histological structure of small and large intestinal mucosa in dogs. The aim of this study was to evaluate the efficacy of budesonide in the treatment of IBD in dogs based on the results of clinical, endoscopic and histopathological examinations.

\section{Materials and Methods}

The experiment was performed on 14 dogs of both sexes and various breeds, aged 6 to 10 years, with body weight of 16 to $25 \mathrm{~kg}$, admitted to the Veterinary Clinic of the University of Warmia and Mazury in Olsztyn. The animals showed symptoms of IBD, including chronic small and large intestinal diarrhea and vomiting of various intensity and frequency. All animals with a suspicion of IBD were subjected to biochemical, radiological, parasitological, bacteriological and mycological stool tests and provocation trials to exclude other diseases with chronic diarrhea. Prior to admission, the patients had been vaccinated against infectious diseases (canine parvovirus type $2-\mathrm{CPV}-2$, canine distemper, canine adenovirus type $2-\mathrm{CAV}-2$, leptospirosis, rabies). Other causes of chronic inflammation of the digestive tract were ruled out, and 14 dogs with severe IBD were qualified for the experiment based on the results of clinical, endoscopic and histopathological analyses of duodenal, jejunal and colonic mucosa. The patients were divided into two groups. Group I comprised 7 dogs administered budesonide (Entocort) at $2 \mathrm{mg} /$ animal/day for 30 days. Group II comprised 7 patients administered placebo (empty gelatin capsule) for 30 days to rule out the influence of the diet on the macroscopic and histopathological changes in small and large intestinal mucosa. Patients from both groups were kept under identical conditions during treatment.

All patients were subjected to clinical examinations to determine their Canine Inflammatory Bowel Disease Activity Index (CIBDAI) scores (Jergens et al. 2003). CIBDAI scores were determined before (day 0) and after treatment (day 30). Endoscopic examinations involving the collection of mucosal samples from the duodenum, jejunum and the colon were performed before (day 0) and after treatment (day 30).

Endoscopic evaluations (panendoscopy and colonoscopy) were performed with the use of the Olympus GIF-Q145 flexible video endoscope with a working length of $1030 \mathrm{~mm}$ and a diameter of $9.8 \mathrm{~mm}$, and the Olympus CF-Q165L video colonoscope with a working length of $1680 \mathrm{~mm}$ and a diameter of 12.8 $\mathrm{mm}$. Biopsy sections were collected from the duodenum, jejunum and the colon with FB-24U-1 biopsy forceps with a diameter of $2.5 \mathrm{~mm}$ and FB-50U-1 forceps with a diameter of $3.7 \mathrm{~mm}$. A total of 18 mucosal samples were collected for histopathological analyses from each patient, including 6 samples from duodenal, jejunal and colonic mucosa each. Extensive macroscopic changes ( 3 points) in the duodenum, jejunum and the colon were observed in all patients. Macroscopic changes were evaluated on a 3-point scale where: 1 point denoted minor changes involving reddening and single extravasations on the surface of duodenal, jejunal and colonic mucosa, 2 points denoted moderate changes involving folding on the surface of duodenal, jejunal and colonic mucosa, minor reddening and infrequent extravasations, and 3 points denoted extensive changes involving significant folding on the surface of duodenal, jejunal and colonic mucosa, considerable reddening and numerous extravasations.

Histopathological evaluations of duodenal, jejunal and colonic mucosa samples were performed before (day 0) and after budesonide treatment (day 30). The analyses were carried out at the Department of Pathological Anatomy, Faculty of Veterinary Medicine, University of Warmia and Mazury in Olsztyn. Sections of intestinal mucosa were evaluated histopathologically in accordance with the recommendations of the WSAVA Gastrointestinal Standardization Group (Day et al. 2008). Changes in the structure of intestinal mucosa were analyzed, and the number of cells in the region of the lamina propria was determined. In all 14 dogs, the observed changes in intestinal sections were classified as extensive ( 3 points on a 3-point scale).

The experiment was conducted with the prior approval of the Local Ethics Committee for Animal Experimentation in Olsztyn pursuant to resolution No. 47/2009/DTN.

\section{Results}

On the completion of treatment, CIBDAI scores in Group I increased in 3 dogs. In 1 dog, the CIBDAI score remained unchanged, and in 3 patients, 
Table 1. Clinical effects changes in CIBDAI scores.

\begin{tabular}{ccccc}
\hline \multirow{2}{*}{ Group } & \multicolumn{5}{c}{ CIBDAI score } \\
\cline { 2 - 5 } & \multicolumn{3}{c}{ Day 0} & \multicolumn{3}{c}{ Day 30 } \\
\cline { 2 - 5 } & $\mathrm{X}$ & $\mathrm{SD}$ & $\mathrm{X}$ & $\mathrm{SD}$ \\
\hline $\mathrm{I}$ & 13.00000 & 1.91485 & 12.14286 & 1.95180 \\
\hline $\mathrm{II}$ & 11.00000 & 1.15470 & $12.28571^{*}$ & 1.70434 \\
\hline
\end{tabular}

$\mathrm{X}-$ mean

SD - standard deviation

CIBDAI scores of Group I and Group II (placebo) patients before (day 0) and after (day 30) budesonide treatment

* significant change at $-\mathrm{p} \leq 005$

** highly significant change at $\mathrm{p} \leq 001$

Table 2. Mean values and standard deviation of endoscopic and histopathological changes in duodenal, jejunal and colonic mucosa on days 0 and 30 in groups I and II.

\begin{tabular}{|c|c|c|c|c|c|c|c|c|}
\hline \multirow{3}{*}{ Group } & \multicolumn{4}{|c|}{ Endoscopic evaluation } & \multicolumn{4}{|c|}{ Histopathological evaluation } \\
\hline & \multicolumn{2}{|c|}{ Day 0} & \multicolumn{2}{|c|}{ Day 30} & \multicolumn{2}{|c|}{ Day 0} & \multicolumn{2}{|c|}{ Day 30} \\
\hline & $\mathrm{X}$ & SD & $\mathrm{X}$ & SD & $\mathrm{X}$ & $\mathrm{SD}$ & $\mathrm{X}$ & $\mathrm{SD}$ \\
\hline I & 2.57143 & 0.53452 & 2.28571 & 0.75593 & 3.00000 & 0.00000 & $1.14286^{*}$ & 0.89974 \\
\hline II & 2.57143 & 0.53452 & 3.00000 & 0.00000 & 3.00000 & 0.00000 & 2.57143 & 0.53452 \\
\hline
\end{tabular}

Key: refer to Table 1

CIBDAI scores were reduced by 2 points $(1 \mathrm{dog})$, 3 points $(1 \mathrm{dog})$ and 5 points $(1 \mathrm{dog})$. After 30 days of budesonide administration, IBD was still evaluated as severe in all patients despite the noted decrease in CIBDAI scores in selected animals. The mean CIBDAI score for Group I dogs was determined at 13.00 before treatment and 12.14 after treatment.

In Group II, the CIBDAI score increased after 30 days of placebo administration. The observed increase resulted from the progression of inflammatory processes in the small and large intestines. After treatment, CIBDAI scores remained unchanged in 2 patients, but increased in 5 dogs - by 1 point $(1 \mathrm{dog})$ or 2 points ( $4 \mathrm{dogs})$. After 30 days of placebo administration, IBD was evaluated as severe in all patients. In Group II, the mean CIBDAI score was determined at 12.29 at the end of the experiment. The increase in CIBDAI scores on day 30 was statistically significant. CIBDAI scores before and after treatment are presented in Table 1.

In Group I, normal macroscopic appearance of intestinal mucosa was not restored in any of the patients after 30 days of budesonide treatment. Macroscopic scores improved by 2 points in 1 dog (from extensive changes, 3 points, to minor changes, 1 point) and by 1 point in 1 patient (from extensive changes, 3 points, to moderate changes, 2 points). In 4 patients, no improvement in the macroscopic appearance of intestinal mucosa was observed after budesonide treatment (moderate changes, 2 points, were observed in 2 dogs, and extensive changes, 3 points, were noted in 2 dogs). The macroscopic appearance of duodenal, jejunal and colonic mucosa deteriorated in 1 individual (significant extravasations, lesions, reddening and folding), and the observed changes were qualified as extensive ( 3 points). In the above animal, the macroscopic picture of colonic mucosa suggested the presence of malignant changes, but these suspicions were not confirmed by histopathological analysis (deep biopsy). The average score after budesonide treatment was determined at 2.29 points.

In Group II fed placebo, no improvement was noted in the macroscopic appearance of duodenal, jejunal and colonic mucosa. Mean and standard deviation of points in this group before treatment was the same as in group I (2.57). In 4 dogs, the pathological changes observed in the intestinal mucosa at the end of the experiment not changed and were still classified as extensive ( 3 points). In 3 animals, the macroscopic appearance of duodenal, jejunal and colonic mucosa deteriorated, and the noted pathological changes were extensive ( 3 points). The mean score after 30 days of placebo administration was determined at 3.00 points. The changes in the macroscopic appearance of duodenal, jejunal and colonic mucosa observed before and after the administration of placebo in Group II are described in Table 2.

Histopathological evaluations of duodenal and 
jejunal sections sampled from all animals revealed lymphocytic-plasmacytic infiltration in the lamina propria, proliferation of connective tissue, variously shaped intestinal villi, congestion and dilation of lymphatic vessels. The observed changes were evaluated as extensive (3 points). The results of a histopathological analysis of colonic mucosa sections from all patients revealed fibrosis and damage to the epithelium, lymphocytic-plasmacytic infiltration in the lamina propria, and proliferation of connective tissue. Severe lymphocytic-plasmacytic enterocolitis (3 points) was noted in all dogs from both groups. After 30 days of budesonide treatment, the intensity of histopathological changes in Group I was significantly reduced to moderate level $(\mathrm{x}-1.14)$. Normal histology was observed in only 2 patients. In the remaining patients from Group I, thin and variously shaped intestinal villi covered with normal epithelium with a large number of goblet cells and intraepithelial lymphocytes were noted after 30 days of budesonide treatment. Numerous lymphocytes and plasmocytes, proliferation of fibrous connective tissue, fluid retention in lymphatic vessels, dilated lymphatic vessels in intestinal villi and vascular congestion were observed in the lamina propria. Mild to moderate lymphocytic-plasmacytic inflammation of the duodenum, jejunum and colon was noted in selected patients. In Group II, no significant improvement in the histological structure of the analyzed intestinal sections was observed after 30 days of placebo administration (x - 2.57). A minor improvement in the histopathological appearance of duodenal, jejunal and colonic mucosa (moderate changes -2 points) was noted in 3 dogs. In the remaining 4 dogs from Group II, the histopathological picture of intestinal sections was not modified, and the observed changes were extensive. Histopathological evaluation in duodenal, jejunal and colonic mucosa before and after treatment are presented in Table 2.

\section{Discussion}

Vomiting and diarrhea did not subside in patients from either group during 30 days of administration of budesonide or placebo. No improvements in vitality, appetite, body gains or ease of digestion, manifested by the excretion of formed stool and uniform stool color, were observed in the examined patients. In Group I, despite the decrease in CIBDAI scores by 2, 3 and 5 points in 3 dogs on day 30 , budesonide treatment was characterized by low clinical efficacy in the treatment of IBD. On the completion of treatment, the average severity of disease was still high in Group I patients, which indicates that budesonide does not constitute effective treatment for severe IBD in dogs. In Group II patients, environmental factors, including diet, did not improve the clinical condition of dogs with IBD. Small and large intestinal diarrhea and chronic vomiting did not subside in any of the patients from Group II. A significant increase in CIBDAI scores was noted after 30 days of placebo administration, which can be attributed to the progression of inflammatory processes in the small and large intestine.

The efficacy of new generation glucocorticosteroids in the treatment of IBD in dogs has been rarely investigated, and very few clinical evaluations can be found in the literature. Pietra et al. (2013) evaluated the therapeutic efficacy of budesonide in 11 dogs with moderate and severe IBD. Each patient was orally administered budesonide (Rafton ${ }^{\circledR}$ ) at $3 \mathrm{mg} / \mathrm{m}^{2}$ every 24 hours, which corresponds to the doses applied in our experiment. CIBDAI scores were determined before the experiment and after 20 days of treatment. The therapeutic efficacy of the drug was evaluated based on the results of clinical evaluations and interviews with the dogs' owners before and after 20 and 30 days of treatment. CIBDAI scores were significantly reduced after 20 days of budesonide administration $(7.4 \pm 3.1$ before treatment, $2.3 \pm 2.1$ after 20 days of treatment). According to the owners, 3 out of 11 dogs $(27 \%)$ receiving budesonide did not show clinical improvement after 30 days of treatment, and large intestinal diarrhea did not subside in these patients. An improvement in the dogs' general behavior, appetite and alertness was reported in 8 animals (73\%). In our study, budesonide did not improve the clinical condition of $57 \%$ of dogs, and in the remaining $43 \%$ of patients, only a minor improvement in CIBDAI scores was noted. Despite statistical differences between groups, the results of our experiment and the cited study indicate that budesonide is not an effective treatment for IBD in dogs.

In Group I, the macroscopic appearance of intestinal mucosa was not restored to norm in any of the patients after 30 days of budesonide treatment. An absence of significant improvement in the macroscopic picture of the small and large intestine was correlated with an absence of clinical improvement. In 4 dogs from Group I, CIBDAI scores increased or remained unchanged on day 30. In Group II dogs which were fed a placebo, no improvement was observed in the macroscopic appearance of duodenal, jejunal and colonic mucosa. On day 30 , the condition of intestinal mucosa deteriorated at the macroscopic level in all Group II animals. The patients' clinical condition did not improve, and their CIBDAI scores increased to 14 points on average at the end of the experiment. In our study, budesonide failed to im- 
prove the macroscopic appearance of small and large intestinal mucosa in dogs with severe IBD (Group I). The observed macroscopic changes in duodenal, jejunal and colonic mucosa were unsatisfactory, which was validated by an absence of improvement in CIBDAI scores in a clinical evaluation.

In Group I, budesonide treatment improved the condition of all patients, but normal histological structure of intestinal mucosa was observed in only 2 animals whose CIBDAI scores decreased by 3 and 5 points. Despite the above, IBD was still evaluated as severe in these patients (CIBDAI score of 10 and 12 points), and the macroscopic appearance of the evaluated intestinal sections improved by 1 point and 2 points. A histopathological evaluation revealed mild to moderate lymphocytic-plasmacytic inflammation of the duodenum, jejunum and colon in the remaining dogs from Group I after 30 days of budesonide treatment. A significant decrease in histopathological scores was observed in Group I patients at the end of the experiment $(\mathrm{x}-1.14)$. In Group II, inflammation of duodenal, jejunal and colonic mucosa was not reduced after 30 days of placebo administration, which indicates that diet and environmental factors had no influence on the outcome of the experiment. In Group II, the results of histopathological analyses were correlated with macroscopic assessments and CIBDAI scores. On day 30, CIBDAI scores increased by 1 point or 2 points in most patients, and IBD was evaluated as severe. In the literature, there are no studies evaluating the influence of budesonide treatment on the histopathological appearance of small and large intestinal mucosa in dogs with IBD. Budesonide was found to be more effective than placebo in the treatment of Crohn's disease in humans, but its therapeutic efficacy was not higher in comparison with other orally administered glucocorticosteroids (Otley and Steinhart 2005). According to Cochrane Reviews, the results of clinical, macroscopic and histopathological evaluations indicate that budesonide is not more effective in maintaining remission in Crohn's disease than prednisone (Papi et al. 2000, Simms and Steinhart 2001). In short-term therapy, budesonide was less effective than conventional steroids, in particular in patients with severe IBD and extensive intestinal changes (Seow et al. 2008). No significant differences were reported in studies comparing the effectiveness of prednisone and budesonide in inducing remission in Crohn's disease (Rutgeerts et al. 1994, Gross et al. 1996, Campieri et al. 1997, Bar-Meir et al. 1998). The therapeutic efficacy of budesonide in the treatment of IBD in humans was validated by the results of this study. The administration of budesonide over a period of 30 days produced only a minor improvement in the clinical condition and macroscopic classification of dogs with IBD. The greatest improvement was noted in the histopathological evaluation, but the extent of the noted changes was moderate. In humans, ulcerative colitis affects only the colonic mucosa, whereas in Crohn's disease, inflammatory processes are noted in all layers of the intestine. In dogs, the disease affects mostly the mucosa of the small and large intestine (the only exception is histiocytic ulcerative colitis in Boxers), which can influence clinical symptoms, macroscopic and histopathological changes, and the kinetics of drug action (Craven et al. 2004, Jergens and Simpson 2012).

The most effective drug for the treatment of IBD should significantly reduce the patients' CIBDAI scores and improve the macroscopic and histopathological appearance of intestinal mucosa. Drugs that improve all three parameters contribute to the efficacy of treatment and prolong remission, which is a very important consideration in the treatment of IBD.

In this study, budesonide was not an effective treatment for IBD in dogs. The tested drug failed to alleviate clinical symptoms of the disease, decrease CIBDAI scores or improve the macroscopic appearance of intestinal mucosa. The highest efficacy of budesonide treatment was observed in the histopathological picture of duodenal, jejunal and colonic mucosa.

\section{Acknowledgments}

This work was supported by the Ministry of Science and Higher Education (Grant No. N N308 234938).

\section{References}

Angelucci E, Malesci A, Danese S (2008) Budesonide: teaching an old dog new tricks for inflammatory bowel disease treatment. Curr Med Chem 15: 2527-2535.

Bar-Meir S, Chowers Y, Lavy A, Abramovitch D, Sternberg A, Leichtmann G, Reshef R, Odes S, Moshkovitz M, Bruck R, Eliakim R, Maoz E, Mittmann U (1998) Budesonide versus prednisone in the treatment of active Crohn's disease. The Israeli Budesonide Study Group. Gastroenterology 115: 835-840.

Campieri M, Ferguson A, Doe W, Persson T, Nilsson LG (1997) Oral budesonide is as effective as oral prednisolone in active Crohn's disease. The Global Budesonide Study Group. Gut 41: 209-214.

Craven M, Simpson JW, Ridyard AE, Chandler ML (2004) Canine inflammatory bowel disease: retrospective analysis of diagnosis and outcome in 80 cases (1995-2002). J Small Anim Pract 45: 336-342. 
Day MJ, Blizer T, Mansell J, Wilcock B, Hall EJ, Jergens A, Minami T, Willard M, Washabau R (2008) Histopathological standards for the diagnosis of gastrointestinal inflammation in endoscopic biopsy samples from the dog and cat: a report from the World Small Animal Veterinary Association Gastrointestinal Standardization Group. J Comp Pathol 138 (Suppl 1): S1-S43.

Gross V, Andus T, Caesar I, Bischoff SC, Lochs H, Tromm A, Schulz HJ, Bär U, Weber A, Gierend M, Ewe K, Schölmerich J (1996) Oral pH-modified release budesonide versus 6-methylprednisolone in active Crohn's disease. German/Austrian Budesonide Study Group. Eur J Gastroenterol Hepatol 8: 905-909.

Jergens AE, Schreiner CA, Frank DE, Niyo Y, Ahrens FE, Eckersall PD, Benson TJ, Evans R (2003) A scoring index for disease activity in canine inflammatory bowel disease. J Vet Intern Med 17: 291-297.

Jergens AE, Simpson KW (2012) Inflammatory bowel disease in veterinary medicine. Front Biosci (Elite Ed) 4: 1404-1419.

Malewska K, Rychlik A, Nieradka R, Kander M (2011) Treatment of inflammatory bowel disease (IBD) in dogs and cats. Pol J Vet Sci 14: 165-171.

Otley A, Steinhart AH (2005) Budesonide for induction of remission in Crohn's disease. Cochrane Database Syst Rev 4: CD000296.

Papi C, Luchetti R, Gili L, Montanti S, Koch M, Capurso $\mathrm{L}$ (2000) Budesonide in the treatment of Crohn's disease: a meta-analysis. Aliment Pharmacol Ther 14: 1419-1428.
Pietra M, Fracassi F, Diana A, Gazzotti T, Bettini G, Peli A, Morini M, Pagliuca G, Roncada P (2013) Plasma concentrations and therapeutic effects of budesonide in dogs with inflammatory bowel disease. Am J Vet Res 74: 78-83.

Rutgeerts P, Löfberg R, Malchow H, Lamers C, Olaison G, Jewell D, Danielsson A, Goebell H, Thomsen OO, Lorenz-Meyer H (1994) A comparison of budesonide with prednisolone for active Crohn's disease. N Engl J Med 331: 842-845.

Seow CH, Benchimol EI, Griffiths AM, Otley AR, Steinhart $\mathrm{AH}$ (2008) Budesonide for induction of remission in Crohn's disease. Cochrane Database Syst Rev 3: CD000296.

Simms L, Steinhart AH (2001) Budesonide for maintenance of remission in Crohn's disease. Cochrane Database Syst Rev 1: CD002913.

Simpson KW, Jergens AE (2011) Pitfalls and progress in the diagnosis and management of canine inflammatory bowel disease. Vet Clin North Am Small Anim Pract 41: 381-398.

Tumulty JW, Broussard JD, Steiner JM, Peterson ME, Williams DA (2004) Clinical effects of short-term oral budesonide on the hypothalamic-pituitary-adrenal axis in dogs with inflammatory bowel disease. J Am Anim Hosp Assoc 40: 120-123. 